EPJ Web of Conferences 41, 04025 (2013)

DOI: $10.1051 /$ epjconf/20134104025

C) Owned by the authors, published by EDP Sciences, 2013

\title{
Ultrafast Non-Thermal Electron Dynamics in Single Layer Graphene
}

D. Brida ${ }^{1}$, C. Manzoni ${ }^{1}$, G. Cerullo ${ }^{1}$, A. Tomadin ${ }^{2}$, M. Polini ${ }^{3}$, R. R. Nair ${ }^{4}$, A. K. Geim ${ }^{4}$, K. S. Novoselov $^{4}$, S. Milana ${ }^{5}$, A. Lombardo ${ }^{5}$, and A. C. Ferrari ${ }^{5}$

${ }^{1}$ IFN-CNR, Dipartimento di Fisica, Politecnico di Milano, Italy

${ }^{2}$ Institute for Quantum Optics and Quantum Information of the Austrian Academy of Sciences, A6020 Innsbruck, Austria

${ }^{3}$ NEST, Istituto Nanoscienze-CNR and Scuola Normale Superiore, I-56126 Pisa, Italy

${ }^{4}$ Department of Physics and Astronomy, University of Manchester, Manchester M13 9 PL, UK

${ }^{5}$ Engineering Department, University of Cambridge, Cambridge, CB3 OFA, UK

\begin{abstract}
We study the ultrafast dynamics of non-thermal electron relaxation in graphene upon impulsive excitation. The 10-fs resolution two color pump-probe allows us to unveil the non-equilibrium electron gas decay at early times.
\end{abstract}

\section{Introduction}

The dynamics of elementary optical excitations in graphene has been the object of a great deal of studies, aimed at understanding the fundamental relaxation mechanisms [1,2]. According to the band structure of graphene (Fig. 1(a)) absorption of a photon of frequency $v$ promotes an electron from an energy $h v / 2$ below the Fermi level in the valence band to an energy hv/2 above the Fermi level in the conduction band. Ultrafast photoexcitation, therefore, creates a strongly non-equilibrium (nonthermal) distribution of electrons in the conduction band (and holes in the valence band), consisting of two peaks at $\pm h v / 2$ with respect to the Fermi level (see Fig. 1(a)).

a)

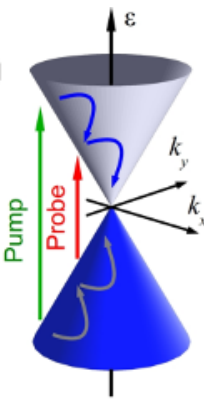

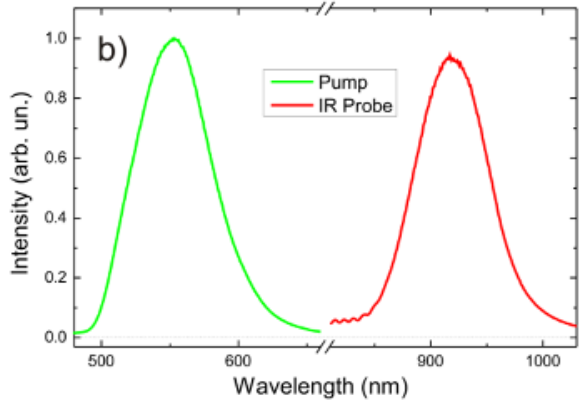

Fig. 1. (a) Sketch of the band structure of a single layer of grapheme and of the pump-probe experiment: the pump pulse excites a non-thermal electronic distribution that is probed at lower energies; (b) pump and probe pulse spectra; (c) $\Delta \mathrm{T} / \mathrm{T}$ time trace at the $860 \mathrm{~nm}$ probe wavelength.

This is an Open Access article distributed under the terms of the Creative Commons Attribution License 2.0, which permits unrestricted use, distribution, and reproduction in any medium, provided the original work is properly cited. 
The primary relaxation process is thermalization due to electron-electron (e-e) interaction, giving rise to an equilibrated Fermi-Dirac (FD) electron distribution with a well defined temperature, higher than that of the lattice. The hot electrons subsequently reach thermal equilibrium with the colder lattice via electron-phonon (e-ph) scattering. These equilibration processes change the transient absorption spectrum over a wide range of energies and an ultrafast timescale. Their time-domain observation calls therefore for the combination of very short pulsewidths and broad spectral tunability.

\section{Experiments}

A large body of experimental work on ultrafast spectroscopy of graphene is available [1-4]. However, so far, most studies have been performed with a time resolution which is too low to observe e-e scattering dynamics and thus have probed an equilibrated hot electron/hole distribution, established within the pump pulse duration $(\sim 100-150 \mathrm{fs})$. These studies have revealed the dynamics of carrier cooling through interaction with the lattice. A number of theoretical studies [5] and indirect experimental evidence [4] however point to an extremely fast e-e relaxation, occurring on the $10-\mathrm{fs}$ timescale. Here we perform pump-probe spectroscopy on graphene using two-color few-opticalcycle pulses. We impulsively excite optical transitions with an ultra-broadband 7-fs pulse centered at $2.25 \mathrm{eV}(2-2.5 \mathrm{eV}$ bandwidth) and probe with a red-shifted 13-fs pulse covering the $1.45-1.2 \mathrm{eV}$ range. The instrumental response function (IRF) of our apparatus (full width at half maximum of the pump-probe cross-correlation) is $\sim 15 \mathrm{fs}[6,7]$. The combination of high time resolution and broad spectral coverage allows capturing the transition from the non-thermal to a FD electron/hole distribution.

Pump-probe experiments are carried out on single-layer graphene films grown by chemical vapor deposition and transferred onto a $100-\mu \mathrm{m}$-thick fused silica substrate. A portion of the substrate is not covered with graphene, thus allowing the measurement of the nonlinear response of the substrate by a simple transverse translation of the sample. In our experiments we observe a prompt rise of the photobleaching signal in the near-IR, that already points out to an ultrafast e-e relaxation, taking place over a timescale comparable to our IRF. Time traces at selected probe wavelengths display a biexponential decay (fig. 1(c)), with a first time constant $\tau 1 \sim 160 \mathrm{fs}$, and a second longer time constant that is not addressed in this study. The first decay is assigned to cooling of the hot electron/hole distribution via interaction with optical phonons, while the longer decay is due to relaxation of the thermalized electron/phonon bath by anharmonic decay of the hot phonons.

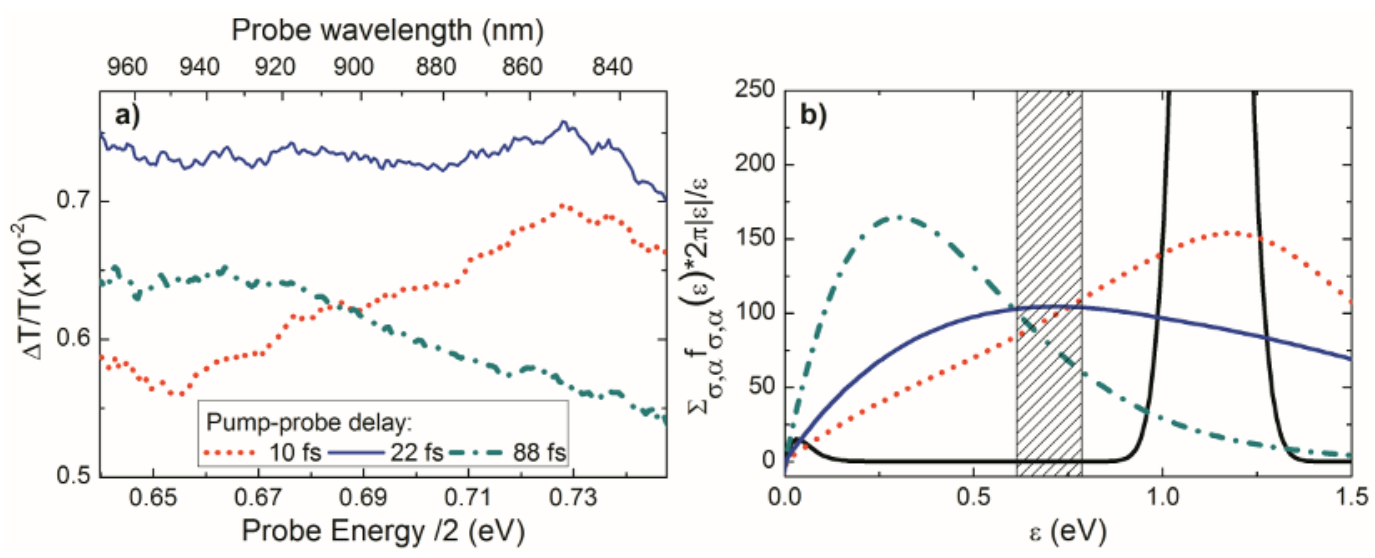

Fig. 2. (a) Transient spectra at selected delays after pumping with a 7-fs visible pulse. The change in slope shows the evolution of the impulsively excited electron distribution to the thermal equilibrium; (b) calculations of the electronic distribution at different delays (thick solid line $\tau_{0}=0$; dotted $\tau_{1}$, dash-dotted $\tau_{2}$ ). The probe window is marked with the shaded area. 
A deeper insight into the dynamical processes leading to e-e thermalization can be obtained by plotting $\Delta \mathrm{T} / \mathrm{T}$ spectra at selected probe delays (Fig. 2(a)). At early times (10 fs), the $\Delta \mathrm{T} / \mathrm{T}$ spectrum has a positive slope, peaking at higher energy; then it progressively flattens ( $22 \mathrm{fs}$ ) and changes to a negative slope, which is retained and then magnified at longer delays.

\section{Results}

To understand the data we recall that the $\Delta \mathrm{T} / \mathrm{T}$ signal for single-layer graphene is the transient electron/hole distribution function induced by the pump and explored at time $\tau$. The sub-10-fs 2.25$\mathrm{eV}$ pump pulse creates an electron distribution peaking at $\sim 1.12 \mathrm{eV}$ above the Fermi level, while the probe pulse interrogates the $0.6-0.72 \mathrm{eV}$ interval.

At early times, therefore, we will observe the tail of this distribution, which has a positive slope. On the other hand for a thermal distribution the $\Delta \mathrm{T} / \mathrm{T}$ spectrum has a negative slope peaking at lower photon energies. The change of slope of the $\Delta \mathrm{T} / \mathrm{T}$ spectrum thus captures the transition from the non-thermal to the thermal regime, which is completed within $\sim 30$ fs. Fig. 2(b) reports a calculation of the transient electronic distribution above Fermi level that shows how, at early times, the electronic distribution (solid line) is still peaked at higher energy and then relaxes to a thermal distribution only at longer times (dash-dotted line). The observed dynamics do not exhibit a significant dependence on the excitation density. By moving from multichannel to single-wavelength detection, we were able to reduce the fluence by a factor of 20 , and saw a substantially unchanged dynamics.

In conclusion, we exploit the very high temporal resolution of our two-color pump-probe scheme to observe the early times in the relaxation dynamics of out-of-equilibrium electrons in single layer graphene. The ultrafast timescale of this process is strongly related to the peculiar band structure and the electronic properties of this fascinating material.

\section{References}

1. D. Sun, Z.-K. Wu, C. Divin, X. B. Li, C. Berger, W. A. de Heer, P. N. First, T. B. Norris, Phys. Rev. Lett. 101, 157402 (2008).

2. B.A. Ruzicka, N. Kumar, S. Wang, K. Ping Loh, H. Zhao, J. Appl. Phys. 109, 084322 (2011).

3. J. Shang, T. Yu, J. Lin, G.G. Gurzadyan, ACS Nano 5, 3278 (2011).

4. M. Breusing, S. Kuehn, T. Winzer, E. Malic, F. Milde, N. Severin, J.P. Rabe, C. Ropers, A. Knorr, T. Elsaesser, Phys. Rev. B 83, 153410 (2011).

5. E. Malic, T. Winzer, E. Bobkin, A. Knorr, Phys. Rev. B 84, 205406 (2011).

6. C. Manzoni, D. Polli and G. Cerullo, Rev. Sci. Instrum. 77, 023103 (2006).

7. D. Brida, C. Manzoni, G. Cirmi, M. Marangoni, S. Bonora, P. Villoresi, S. De Silvestri and G. Cerullo, J. Opt. 12, 013001 (2010). 